\title{
A STEADY-STATE HEAT CONDUCTION PROBLEM OF A NONHOMOGENOUS CONICAL BODY
}

\author{
I. ECsedi, A. BAKsa \\ Institute of Applied Mechanics, University of Miskolc \\ 3515 Miskolc-Egyetemváros, Hungary \\ istvan.ecsedi@uni-miskolc.hu attila.baksa@uni-miskolc.hu
}

[Received: April 14, 2021; Accepted: June 22, 2021]

\begin{abstract}
Numerous studies and textbooks deal with the steady-state thermal conduction of radially nonhomogeneous circular cylinder. In contrast, there are relatively few studies on the thermal conduction problems of conical solid bodies. This study is intended as a modest contribution to the solution of thermal conductance problems of nonhomogeneous conical bodies. A one-dimensional steady-state heat conduction in nonhomogeneous conical body is considered. The thermal conductivity of the hollow conical body in a suitable chosen spherical coordinate system depends on the polar angle but is independent of the radial coordinate and azimuthal angle coordinate. A functionally graded type of material inhomogeneity is considered. All results of the paper are based on Fourier's theory of heat conduction in solid bodies.
\end{abstract}

Mathematical Subject Classification: 80A20

Keywords: heat conduction, steady-state, conical body, nonhomogenous, functionally graded material

\section{INTRODUCTION}

Numerous studies and textbooks of heat transfer deal with the steady-state thermal conduction in radially nonhomogeneous circular cylinder 1-3]. A lot of papers consider the functionally graded material inhomogeneity of solid bodies. Functionally graded materials (FGMs) are new advanced heat resisting materials used in modern technologies [4, 5]. The basic concept is to mix the ceramic and metal such that the material properties continuously vary from one constituent material to other. The coefficients of governing equations for temperature distribution are coordinate dependent, as the thermal properties are function of position.

In an important paper by Porchaloempong et al. [6] a two-dimensional energy balance approach was used to model the temperature distribution in conduction heated conically shaped bodies. The numerical solution was based on finite differences. The cone was divided into small volume elements. The inner elements were concentric rings of rectangular cross sections while those at the side surfaces had trianglular cross sections. Energy balance equations for volume elements were solved explicitly. The conduction heat transfer model developed in this study can accurately predict the temperature distribution in a conically shaped container [6]. The paper [7] presents an 
analytical solution for heat transfer in heterogeneous conical shells with temperature dependent conduction coefficients. The geometry of the shell is completely conically shaped. The heat transfer equation should be first transformed using the Kirchhoff transformation 7]. The obtained differential equation is solved by the Green function [7]. An exact analytical solution for anisotropic conductive heat transfer in composite conical shells is presented by Norouzi and Rahmani [8]. The shell has a full conical shape and the fibers are winded around the conical body. The exact solution of the temperature field is obtained via the separation of variables method. The developed analytical solution is validated by a numerical solution [8].

This study is intended as a modest contribution to the solution of the thermal conductance problem of a hollow conical body made of functionally graded material. The thermal conductivity in a suitable chosen spherical coordinate system depends on the polar angle but it is independent of the radial coordinate and azimuthal angle coordinate. Two types of the polar-nonhomogeneity are considered which are the power nonhomogeneity and the exponential nonhomogeneity [4, 5].

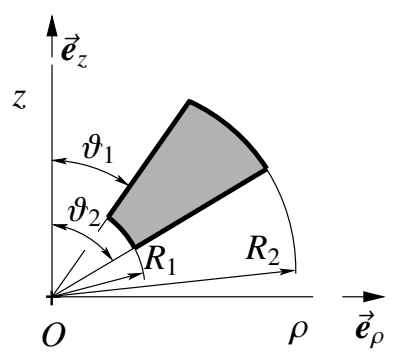

Figure 1. Meridian section of the conical body

Figure 1 shows the meridian section of the hollow conical body which is bordered by two conical and two spherical surfaces. The definition of the spherical coordinates $r, \varphi, \vartheta$ are given in Figure 2 .

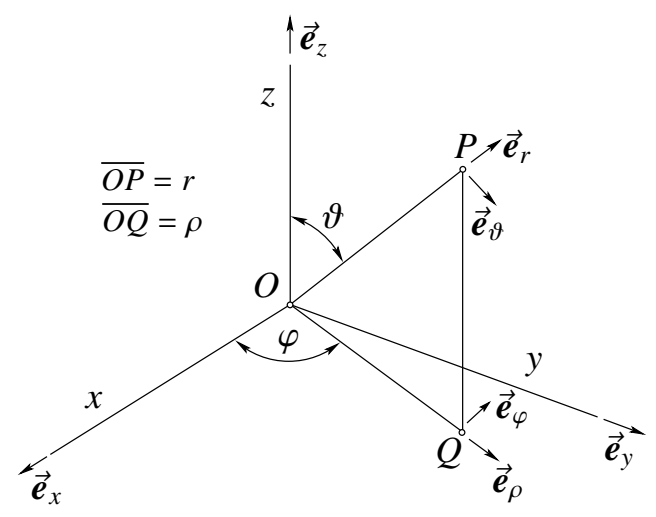

Figure 2. Spherical coordinate system 
The space domain occupied by the conical body is denoted by $B$

$$
B=\left\{(r, \varphi, \vartheta) \mid R_{1} \leq r \leq R_{2}, 0 \leq \varphi \leq 2 \pi, 0 \leq \vartheta_{1} \leq \vartheta_{2} \leq \pi\right\} .
$$

The boundary conditions for the considered steady-state heat conduction are

$$
\begin{gathered}
\frac{\partial T}{\partial r}=0 \quad r=R_{1} \quad 0 \leq \varphi \leq 2 \pi \quad \vartheta_{1} \leq \vartheta \leq \vartheta_{2}, \\
\frac{\partial T}{\partial r}=0 \quad r=R_{2} \quad 0 \leq \varphi \leq 2 \pi \quad \vartheta_{1} \leq \vartheta \leq \vartheta_{2}, \\
-\frac{k(\vartheta)}{h_{1}} \frac{\partial T}{\partial \vartheta}+T=t_{1} \quad \vartheta=\vartheta_{1} \quad 0 \leq \varphi \leq 2 \pi \quad R_{1} \leq r \leq R_{2}, \\
\frac{k(\vartheta)}{h_{2}} \frac{\partial T}{\partial \vartheta}+T=t_{2} \quad \vartheta=\vartheta_{2} \quad 0 \leq \varphi \leq 2 \pi \quad R_{1} \leq r \leq R_{2} .
\end{gathered}
$$

In equations $(1.2)-1.5) T=T(r, \varphi, \vartheta)$ is the temperature field of the nonhomogeneous conical body, $k=k(\vartheta)$ is the thermal conductance, $h_{1}$ and $h_{2}$ are the heat transfer coefficients and $h_{i}=$ constants $(i=1,2)$. The temperatures of surrounding medium at the inner and outer conical boundary surfaces are denoted by $t_{1}=$ constant, $t_{2}=$ constant, they are given. The boundary conditions 1.2 and (1.3) express that, the spherical surfaces at $r=R_{1}$ and $r=R_{2}$ are thermal insulated surfaces $[1,3,9]$.

In the present problem according to the Fourier's theory of heat conduction in a solid nonhomogeneous body we have

$$
\begin{aligned}
\boldsymbol{q}=-k(\vartheta) \nabla T \quad \nabla & =\frac{\partial}{\partial r} \boldsymbol{e}_{r}+\frac{1}{r \sin \vartheta} \frac{\partial}{\partial \varphi} \boldsymbol{e}_{\varphi}+\frac{1}{r} \frac{\partial}{\partial \vartheta} \boldsymbol{e}_{\vartheta}, \\
\nabla \cdot \boldsymbol{q} & =0 \quad(r, \varphi, \vartheta) \in B .
\end{aligned}
$$

The unit vectors of spherical coordinate system $\operatorname{Or} \varphi \vartheta$ are $\boldsymbol{e}_{r}, \boldsymbol{e}_{\varphi}$ and $\boldsymbol{e}_{\vartheta}$ (Fig. 2), and in equations $(1.7)$ and $(1.8)$ the dot denotes the scalar product of two vectors. The heat flux vector is denoted by $\boldsymbol{q}$. The substitution of equation 1.6 ) into equation (1.8) gives 10

$$
\begin{aligned}
& \nabla \cdot(k(\vartheta) \nabla T)=k(\vartheta) \triangle T+\nabla k \cdot \nabla T=k(\vartheta)\left[\frac{1}{r^{2}} \frac{\partial}{\partial r}\left(r^{2} \frac{\partial T}{\partial r}\right)+\right. \\
& \left.\frac{1}{r^{2} \sin \vartheta} \frac{\partial}{\partial \vartheta}\left(\sin \vartheta \frac{\partial T}{\partial \vartheta}\right)+\frac{1}{r^{2} \sin ^{2} \vartheta} \frac{\partial^{2} T}{\partial \varphi^{2}}\right]+\frac{1}{r^{2}} \frac{\partial k}{\partial \vartheta} \frac{\partial T}{\partial \vartheta}=0 \quad(r, \varphi, \vartheta) \in B .
\end{aligned}
$$

We assume that $T=T(\vartheta)$, that is the temperature field depends on only the polar angle $\vartheta$. This assumption leads to an ordinary differential equation for $T=T(\vartheta)$

$$
\frac{k(\vartheta)}{\sin \vartheta} \frac{\mathrm{d}}{\mathrm{d} \vartheta}\left(\sin \vartheta \frac{\mathrm{d} T}{\mathrm{~d} \vartheta}\right)+\frac{\mathrm{d} k}{\mathrm{~d} \vartheta} \frac{\mathrm{d} T}{\mathrm{~d} \vartheta}=0, \quad \vartheta_{1}<\vartheta<\vartheta_{2}
$$

It is obvious that $T=T(\vartheta)$ satisfies the two-point boundary conditions

$$
-\frac{k\left(\vartheta_{1}\right)}{h_{1}}\left(\frac{\mathrm{d} T}{\mathrm{~d} \vartheta}\right)_{\vartheta=\vartheta_{1}}+T_{1}=t_{1}, \quad T_{1}=T\left(\vartheta_{1}\right),
$$




$$
\frac{k\left(\vartheta_{2}\right)}{h_{2}}\left(\frac{\mathrm{d} T}{\mathrm{~d} \vartheta}\right)_{\vartheta=\vartheta_{2}}+T_{2}=t_{2}, \quad T_{2}=T\left(\vartheta_{2}\right) .
$$

\section{Solution of the heAT CONDUCTION BOUndARY-VAlue PROBlem}

The general solution of a second order ordinary differential equation 1.9 can be represented as 10

$$
T(\vartheta)=C_{1} \int_{\vartheta_{1}}^{\vartheta} \frac{\mathrm{d} \omega}{k(\omega) \sin \omega}+C_{2}, \quad \vartheta_{1} \leq \vartheta \leq \vartheta_{2},
$$

where $C_{1}$ and $C_{2}$ are arbitrary constants whose values can be determined by the use of boundary conditions $(1.10)$ and $(1.11)$. We give the expressions of the constants of integration in terms of $T_{1}=T\left(\vartheta_{1}\right)$ and $T_{2}=T\left(\vartheta_{2}\right)$

$$
C_{1}=\frac{T_{2}-T_{1}}{\int_{\vartheta_{1}}^{\vartheta_{2}} \frac{\mathrm{d} \vartheta}{k(\vartheta) \sin \vartheta}}, \quad C_{2}=T_{1} .
$$

The temperature field in the polar-nonhomogeneous conical body in terms of $T_{1}$ and $T_{2}$ is as follows

$$
T(\vartheta)=T_{1}+\left(T_{2}-T_{1}\right) \frac{\int_{\vartheta_{1}}^{\vartheta} \frac{\mathrm{d} \omega}{k(\omega) \sin \omega}}{\int_{\vartheta_{1}}^{\vartheta_{2}} \frac{\mathrm{d} \vartheta}{k(\vartheta) \sin \vartheta}}, \quad \vartheta_{1} \leq \vartheta \leq \vartheta_{2},
$$

and we have

$$
k(\vartheta) \frac{\mathrm{d} T}{\mathrm{~d} \vartheta}=\frac{T_{2}-T_{1}}{\int_{\vartheta_{1}} \frac{\mathrm{d} \vartheta}{k(\vartheta) \sin \vartheta}} .
$$

Formula 2.4 shows that $T=T(\vartheta)$ increases monotonically in $\vartheta_{1} \leq \vartheta \leq \vartheta_{2}$, if $T_{2}>T_{1}$ and $T=T(\vartheta)$ is monotonic decrease in $\vartheta_{1}<\vartheta<\vartheta_{2}$ if $T_{1}>T_{2}$.

Substitution of equations $(2.3)$ and $(2.4)$ into equation 1.10, , 1.11 yields a system of linear equations for the unknown boundary temperature $T_{1}$ and $T_{2}$. The solution of this system of linear equations can be represented as

$$
\begin{aligned}
& T_{1}=t_{1} \frac{1+a_{2}}{1+a_{1}+a_{2}}+t_{2} \frac{a_{1}}{1+a_{1}+a_{2}}, \\
& T_{2}=t_{1} \frac{a_{2}}{1+a_{1}+a_{2}}+t_{2} \frac{1+a_{1}}{1+a_{1}+a_{2}} .
\end{aligned}
$$

In equations (2.5) and (2.6)

$$
a_{1}=\frac{1}{h_{1} \sin \vartheta_{1} \int_{\vartheta_{1}}^{\vartheta_{2}} \frac{\mathrm{d} \vartheta}{k(\vartheta) \sin \vartheta}}, \quad a_{2}=\frac{1}{h_{2} \sin \vartheta_{2} \int_{\vartheta_{1}}^{\vartheta_{2}} \frac{\mathrm{d} \vartheta}{k(\vartheta) \sin \vartheta}} .
$$


It is evident, that

$$
\lim _{h_{i} \rightarrow \infty} a_{i}=0 \quad(i=1,2),
$$

then the Robin's type boundary conditions 1.10 and 1.11 are replaced by Dirichlet type boundary conditions that is

$$
\text { for } h_{i}=\infty \quad T\left(\vartheta_{i}\right)=t_{i} \quad(i=1,2) .
$$

\section{EXAMPLES}

3.1. Homogeneous conical body. For a homogeneous conical body $k=k_{0}=$ constant. In this case

$$
T(\vartheta)=T_{1}+\left(T_{2}-T_{1}\right) \frac{\ln \left|\frac{\tan \frac{\vartheta}{2}}{\tan \frac{\vartheta_{1}}{2}}\right|}{\ln \left|\frac{\tan \frac{\vartheta_{2}}{2}}{\tan \frac{\vartheta_{1}}{2}}\right|} .
$$

and

$$
a_{i}=\frac{k_{0}}{h_{i} \sin \vartheta_{i} \ln \left|\frac{\tan \frac{\vartheta_{2}}{2}}{\tan \frac{\vartheta_{1}}{2}}\right|} \quad(i=1,2) .
$$

It is worth noticing that if $\delta=\vartheta_{2}-\vartheta_{1}<<\vartheta_{1}$ and $\varepsilon=\vartheta-\vartheta_{1}$ then

$$
\begin{gathered}
\sin \vartheta=\sin \left(\vartheta_{1}+\varepsilon\right)=\sin \vartheta_{1} \cos \varepsilon+\cos \vartheta_{1} \sin \varepsilon \approx \sin \vartheta_{1}+\varepsilon \cos \vartheta_{1}, \\
\frac{1}{\sin \vartheta} \approx \frac{1}{\sin \vartheta_{1}\left(1+\varepsilon \frac{\cos \vartheta_{1}}{\sin \vartheta_{1}}\right)} \approx \frac{1}{\sin \vartheta_{1}\left[1+\left(\vartheta-\vartheta_{1}\right) \frac{\cos \vartheta_{1}}{\sin \vartheta_{1}}\right]} \approx \\
\frac{1}{\sin \vartheta}\left[1-\left(\vartheta-\vartheta_{1}\right) \frac{\cos \vartheta_{1}}{\sin \vartheta_{1}}\right], \\
\int_{\vartheta_{1}}^{\vartheta} \frac{\mathrm{d} \vartheta}{\sin \vartheta} \approx \frac{1}{\sin \vartheta_{1}}\left(\vartheta-\vartheta_{1}\right) .
\end{gathered}
$$

Substitution of equation (3.5) into equation 2.3 gives an approximation formula for the temperature distribution across the thickness of thin-walled conical tube

$$
T(\vartheta)=T_{1}+\left(T_{2}-T_{1}\right) \frac{\vartheta-\vartheta_{1}}{\vartheta_{2}-\vartheta_{1}}
$$




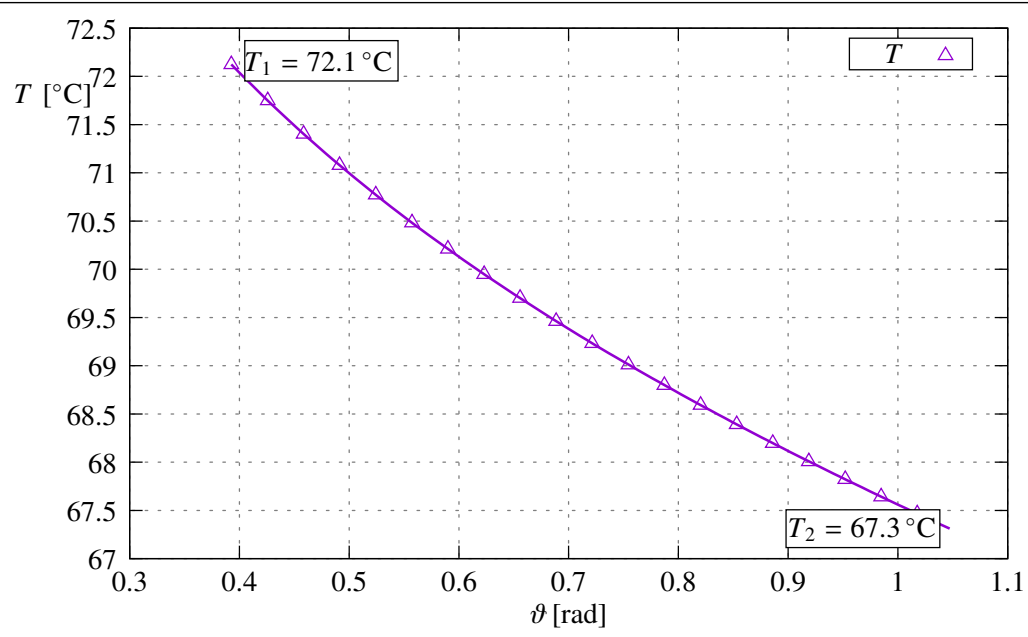

Figure 3. Illustration of temperature in a conical body as a function of $\vartheta$

Figure 3 shows the temperature as a function of polar angle $\vartheta$ for the homogeneous conical body, if

$$
\begin{gathered}
t_{1}=298 \mathrm{~K}=25^{\circ} \mathrm{C}, \quad t_{2}=393 \mathrm{~K}=120^{\circ} \mathrm{C}, \quad \vartheta_{1}=\frac{\pi}{8}, \quad \vartheta_{2}=\frac{\pi}{3}, \quad R_{1}=0.4 \mathrm{~m}, \\
R_{2}=2 \mathrm{~m}, \quad k_{0}=81.1 \frac{\mathrm{W}}{\mathrm{Km}}, \quad h_{1}=20 \frac{\mathrm{W}}{\mathrm{Km}^{2}}, \quad h_{2}=10 \frac{\mathrm{W}}{\mathrm{Km}^{2}} .
\end{gathered}
$$

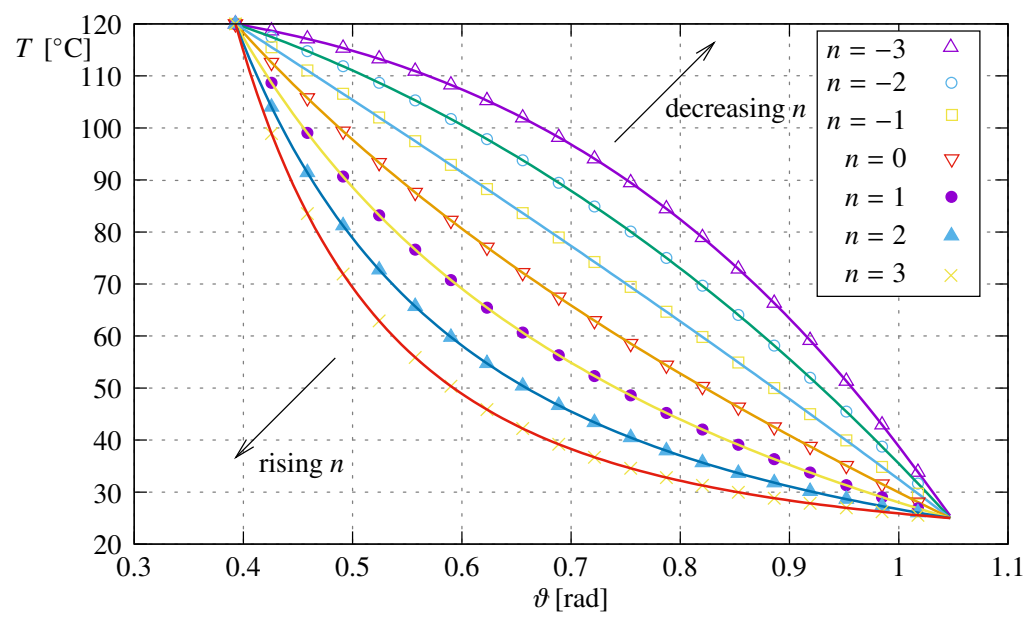

Figure 4. Temperature distribution in conical body for seven different values of power index $n$ 
3.2. Power law nonhomogeneity. In this case the thermal nonhomogeneity has the form

$$
k(\vartheta)=k_{0}\left(\frac{\vartheta}{\vartheta_{1}}\right)^{n} \quad k_{0}=\text { constants, } \quad \vartheta_{1} \leq \vartheta \leq \vartheta_{2},
$$

where $n$ is the power index. The applied thermal boundary conditions at $\vartheta=\vartheta_{1}$ and at $\vartheta=\vartheta_{2}$ are Dirichlet's type, the inner and outer boundary conical surfaces have prescribed temperatures. The plots of $T=T(\vartheta, n)$ for seven different values of power index $n(n=-3,-2,-1,0,1,2,3)$ are shown in Figure 4 if

$$
\vartheta_{1}=\frac{\pi}{8}, \quad \vartheta_{2}=\frac{\pi}{3}, \quad R_{1}=0.4 \mathrm{~m}, \quad R_{2}=0.8 \mathrm{~m}, \quad k_{0}=81.1 \frac{\mathrm{W}}{\mathrm{Km}}, .
$$

3.3. Exponential nonhomogeneity. In this example the same data are used as in Examples 3.2 except $k=k(\vartheta)$. The polar-nonhomogeneity of the exponentially graded hollow conical body is

$$
k(\vartheta)=k_{0} \exp (\beta \vartheta) \quad \vartheta_{1} \leq \vartheta \leq \vartheta_{2} .
$$

In equation (3.8) $\beta$ is a material property [4, 5]. The plots of the function $T=T(\vartheta, \beta)$ against $\vartheta$ for seven different values of graded parameter $\beta$ are shown in Figure 5 $(\beta=-4.0,-2.5,-1.0,0.0,1.0,2.5,4.0)$.

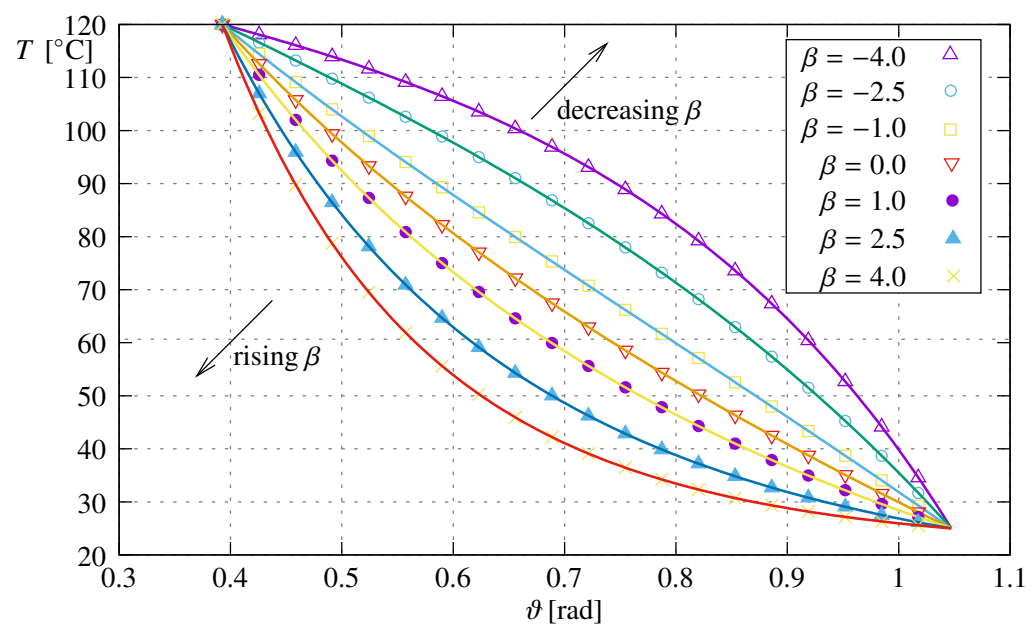

Figure 5. Temperature distribution in conical hollow body for seven different values of power index $\beta$

\section{Overall heat transfer COEFFicient.}

The overall heat transfer coefficient in steady state heat conduction problem is an important structural property of a solid body in which the heat is flowing between the two separated parts of its boundary surfaces. From the higher temperature boundary part of the body to the lower temperature boundary part of the body the process of 
heat flow is characterized by the overall heat transfer coefficient according to equation (4.1)

$$
Q=\Lambda\left(T_{2}-T_{1}\right), \quad T_{2}>T_{1},
$$

where $Q$ is the heat flow in unit time, $T_{1}$ and $T_{2}$ are given temperatures and $\Lambda$ is the overall heat transfer coefficient.

In the present problem, the heat flow between the conical surfaces $\partial B_{1}$ and $\partial B_{2}$

$$
\begin{aligned}
& \partial B_{1}=\left\{(r, \varphi, \vartheta) \mid R_{1} \leq r \leq R_{2}, 0 \leq \varphi \leq 2 \pi, \vartheta=\vartheta_{1}\right\}, \\
& \partial B_{2}=\left\{(r, \varphi, \vartheta) \mid R_{1} \leq r \leq R_{2}, 0 \leq \varphi \leq 2 \pi, \vartheta=\vartheta_{2}\right\},
\end{aligned}
$$

can be obtained from the equation 4.4

$$
Q=-\int_{\partial B_{1}} \frac{k(\vartheta)}{r} \frac{\mathrm{d} T}{\mathrm{~d} \vartheta} \mathrm{d} A .
$$

The area element of the boundary surface segment $\partial B_{1}$ is

$$
\mathrm{d} A=2 \pi r \sin \vartheta_{1} \mathrm{~d} r
$$

Substitution of equation 2.3 and equation 4.5 into the formula of heat flow $Q$ (4.4) gives

$$
Q=\left(T_{2}-T_{1}\right) \frac{2 \pi\left(R_{2}-R_{1}\right)}{\int_{\vartheta_{2}} \frac{\mathrm{d} \vartheta}{k(\vartheta) \sin \vartheta}} .
$$

From equations 4.1 and 4.6 it follows that

$$
\Lambda=\frac{\int_{\vartheta_{1}}^{\vartheta_{2}} \frac{\mathrm{d} \vartheta}{k(\vartheta) \sin \vartheta}}{2 \pi\left(R_{2}-R_{1}\right)} .
$$

The effect of nonhomogeneity on the overall heat transfer coefficient will be analysed for two types of polar nonhomogeneity. In both cases, the following data are used:

$$
\vartheta_{1}=\frac{\pi}{8}, \quad \vartheta_{2}=\frac{\pi}{3}, \quad R_{1}=0.4 \mathrm{~m}, \quad R_{2}=2 \mathrm{~m}, \quad k_{0}=81.1 \frac{\mathrm{W}}{\mathrm{Km}} .
$$

4.1. Power nonhomogeneity. In this case

$$
k(\vartheta)=k_{0}\left(\frac{\vartheta}{\vartheta_{1}}\right)^{n} \quad-4 \leq n \leq 4 .
$$

The plot of the function $\Lambda=\Lambda(n)$ against $n$ is presented in Figure6. The relation between $\Lambda(-4)$ and $\Lambda(4)$ can be considered a kind of degree of the power nonhomogeneity

$$
\frac{\Lambda(-4)}{\Lambda(4)}=54.9186
$$




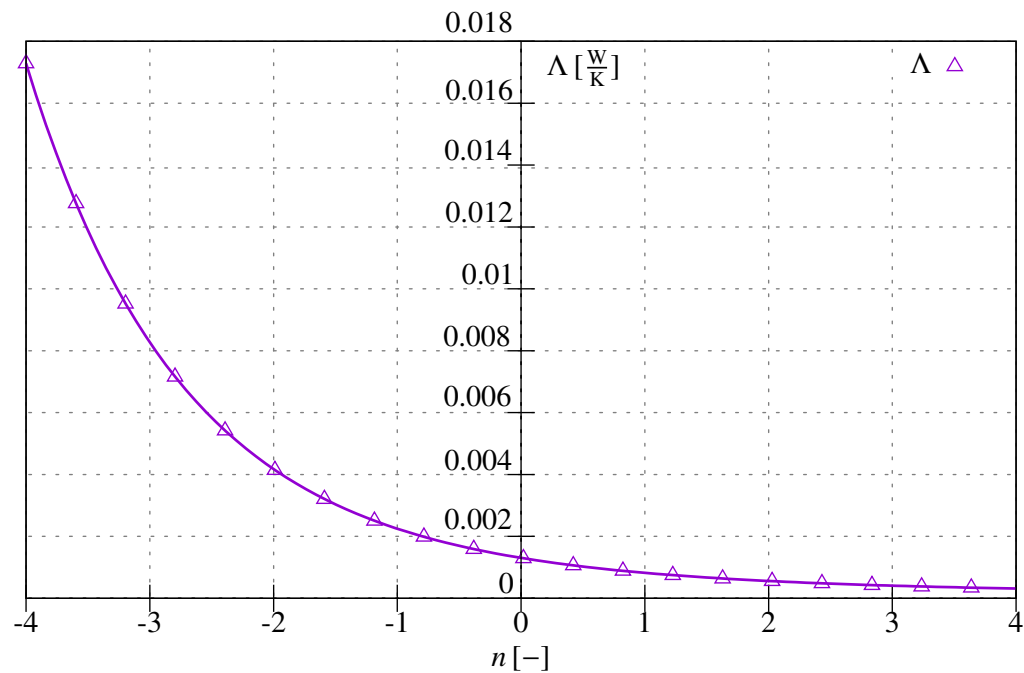

Figure 6. The overall heat transfer coefficient as a function of power index $n$ of inhomogeneity

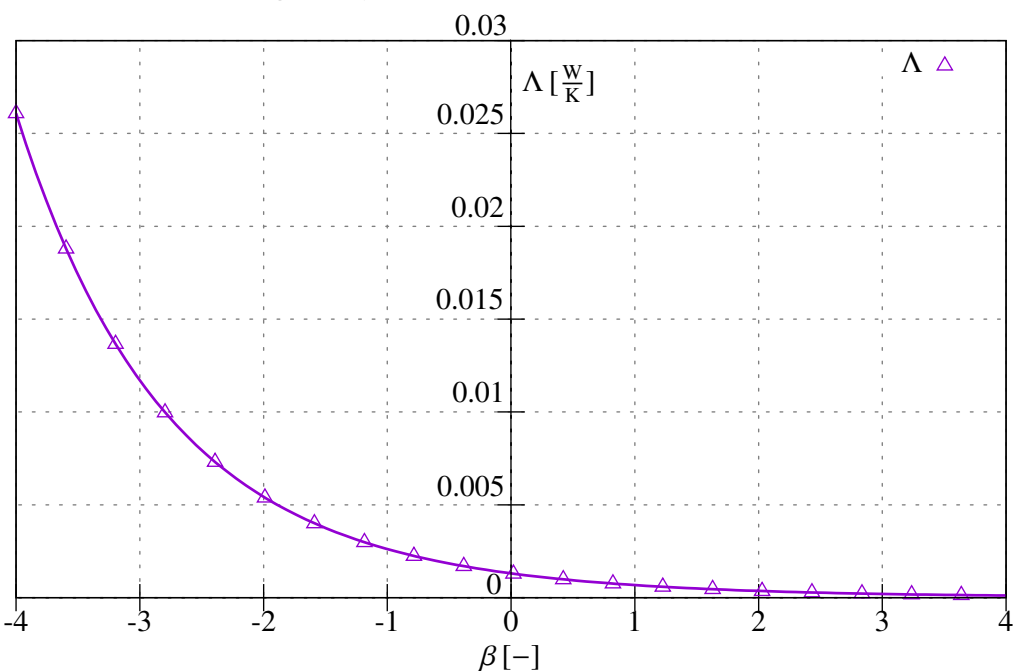

Figure 7. The overall heat transfer coefficient as a function of inhomogeneity parameter $\beta$

4.2. Exponentially nonhomogeneity. The exponentially nonhomogeneity of the polar-nonhomogeneous hollow conical body is described by equation 4.11

$$
k(\vartheta)=k_{0} \exp (\beta \vartheta) \quad-4 \leq \beta \leq 4 .
$$

The plot of the function $\Lambda=\Lambda(\beta)$ is shown in Figure 7 for $-4 \leq \beta \leq 4$. The kind of degree of exponentially nonhomogeneity can be characterized by the ratio of $\Lambda(-4)$ 
to $\Lambda(4)$. In the present problem

$$
\frac{\Lambda(-4)}{\Lambda(4)}=230.97
$$

\section{Conclusions}

A one-dimensional steady-state heat conduction problem of a nonhomogenous hollow conical body is solved. The considered conical body is bordered by two conical surfaces and two spherical surfaces. The thermal conductance depends on the polar angle in a suitably chosen spherical coordinate system. Two-types of the polar-nonhomogeneity are considered. Firstly, the power nonhomogeneity is analyzed and secondly the exponentially nonhomogeneity is considered. For both cases the overall heat transfer coefficient is computed. The presented analytical solution of the temperature field can be used in the solution of stationary thermoelastic problems, when the temperature field separates from the elastic field. In this case the elastic and the temperature fields are uncoupled. Another possible application of the numerical results of this paper is to verify the accuracy of the usual numerical methods such as FEM, finite-difference method, boundary element method, etc.

\section{REFERENCES}

1. H. S. Carslaw and J. C. Jaeger. Conduction of Heat in Solids. London, Oxford, 1947.

2. M. N. Ozisik. Boundary Value Problmes of Heat Conduction. Scranton, Ps., International Textbook, 1968.

3. S. Whitaker. Foundamental Principles of Heat Transfer. Pergamon Press Inc., New York, 2013.

4. S. Suresh and A. Mortensen. Fundamentals of Functionally Graded Materials. IOM Communications, New Yersy, 2003.

5. Y. Miyamoto, W. A. Kaysser, and B. H. Robin. Functionally Grade Materials: Deisgn, Proessing and Applications. Boston, UK Kluwer Academic Publishers, 1999.

6. P. Pornchaloempong, M. O. Balaban, K. V. Chan, and A. A. Teixera. "Numerical solution of conduction heating in conical shape bodies." Journal of Food Process Engineering, 25, (2003), pp. 539-555. DOI: $10.1111 /$ j. 1745-4530 . 2003 . tb00650.x

7. B. E. Manesh, M. M. Shahmardoan, and M. Norouzi. "Analytical solution of heat transfer in heterogeneous composite coniceal shells with temperature dependent coefficients." Amirkabir Journal of Mechanical Engineering 529, (2020), pp. 639 642. DOI: $10.22060 / \mathrm{mej} \cdot 2019.15168 .6050$.

8. M. Norowzi and H. Rahmani. "On exact solution for anisotropic heat conduction in composite conical shells." International Journal of Thermal Stresses 94, (2015), pp. 110-125. DOI: 10.1016/j.ijthermalsci.2015.02.018. 
A steady-state heat conduction problem of a nonhomogeneous conical body 97

9. B. Sunddén. Introduction to Heat Transfer. WIT Press, Southampton, Boston, 2012.

10. G.A. Korn and T.M. Korn. Mathematical Handbook for Scientists and Engineergs. New York: D von Nostrand, 1961. 